DEMONSTRATIO MATHEMATICA

Vol. XXV No $4 \quad 1992$

Peter Burmeister, Boleslaw Wojdylo

THE MEANING OF BASIC CATEGORY THEORETICAL NOTIONS

IN SOME CATEgORIES OF PARTIAL ALgebras. II

PRODUCTS AND COPRODUCTS

\title{
Introduction
}

This paper is a direct continuation of the paper [4], to which we refer the reader for the definitions of basic concepts and some preliminary results. Observe that also the numeration of the statements and figures is continued.

For concepts not defined here we refer the reader in particular to [4] and [2], however see also [1] or [3] as far as partial algebras are concerned, and [5] or [7] with respect to category theoretical concepts. A great part of the results and proofs presented here can already be found in the report [8], which has never been published so far.

\section{The main theorems (continuation)}

We start with two remarks:

Remark 1. Since we often have to treat the behaviour of our different kinds of morphisms with respect to constants, we collect here the following observations, where $f: \mathbb{A} \rightarrow \mathbb{B}$ is assumed to be a morphism in any of our categories, and the type $\tau$ is to specify at least one nullary operation symbol, say $\varphi$, in $\Omega$.

(i) If $f$ is a homomorphism, then the existence of $\varphi^{\mathbb{A}}$ implies the one of $\varphi^{\mathbb{B}}$, while the existence of $\varphi^{\mathbb{B}}$ does not influence the one of $\varphi^{\mathbb{A}}$.

(ii) If $\mathbf{f}$ is a closed homomorphism, then $\varphi^{\mathbb{A}}$ exists, if and only if $\varphi^{\mathbb{B}}$ exists.

(iii) If $f$ is a quomorphism, then the existence of $\varphi^{\mathbb{A}}$ only 
implies the existence of $\varphi^{B}$, if $\varphi^{A}$ belongs to the domain of $f_{i}$ and the existence of $\varphi^{\mathbb{B}}$ does not influence at all the one of $\varphi^{A}$.

(iv) If $f$ is a closed quomorphism, then the existence of $\varphi^{\mathbb{B}}$ always implies the existence of $\varphi^{\mathbb{A}}$ and that $\varphi^{\mathbb{A}}$ belongs to the domain of $f$. However, the existence of $\varphi^{\mathbb{A}}$ implies the one of $\varphi^{\mathbb{B}}$ only, if $\varphi^{A}$ belongs to the domain of $f$. Or, formulated in a different way: if $\varphi^{B}$ does not exist, then either $\varphi^{\mathbb{A}}$ does not exist, or it does not belong to the domain of $f$.

(v) If $f$ is a conformism, then the existence of $\varphi^{B}$ always implies the one of $\varphi^{A}$, while the existence of $\varphi^{A}$ has no influence on the one of $\varphi^{\mathbb{B}}$.

Remark 2. Before we discuss the existence of products and their eventual structure - in our five categories, let us observe that in $\mathbb{E}-$ Quom $\left((1)_{\varphi \in \Omega}\right)$ and in $\operatorname{Eonf}\left((1)_{\varphi \in \Omega}\right)$ we shall meet a situation, where the objects "explode" in a similar way as we already have observed it in connection with terminal objects in E-jom ((1) ( $_{\varphi \in \Omega}$ ) (cf. [4], Theorem 1) - and the new situation even proves to be similar to the earlier one.

In order to see what may happen let us consider the partial algebra $A$ depicted in Figure 3 as an object of (E-Lum((1)) respectively of Conf ((1)), and we want to investigate a possible product object of a product of $\mathbb{A}$ with $\mathbb{A}$ in one of these categories. For $[-2 u o m((1))$ we consider the partial algebra $\mathbb{P}=\left(\mathbb{P}, \varphi^{\mathbb{P}}\right)$ as depicted in Figure 3 by the solid arrows (representing the fundamental operation $\varphi^{\mathbb{P}}$ ), while for Conf $((1))$ we consider the partial algebra $P^{\prime}=\left(P^{\prime}, \varphi^{P^{\prime}}\right)$ depicted in Figure 3 with the solid as well as with the broken arrows representing instances of $\varphi^{\mathbb{P}^{\prime}}$. Only part of $\mathbb{P}$ respectively $\mathbb{P}^{\prime}$ is shown, and elements belonging only to $P^{\prime}$ have smaller labels. Moreover, we have partial mappings $\mathbf{p}_{1}, \mathbf{p}_{2}: \mathbf{P}-\rightarrow \mathbf{A}$ and $\mathbf{p}_{1}^{\prime}, \mathbf{p}_{2}^{\prime}: \mathbf{P}^{\prime}-\rightarrow \mathbf{A}$, and for an element $\mathbf{q \in P}$ or $q \in \mathbf{P}^{\prime}$ the label means $\left(p_{1}(q), p_{2}(q)\right)$ respectively $\left(p_{1}^{\prime}(q), p_{2}^{\prime}(q)\right)$, "-" stands for "undefined". One has infinitely many elements with the same label (elements occurring with broken arrows only belong to $\left.P^{\prime}\right)$. The "local structure" ending at points labeled by $(c, a)$ or $(b, c)$ has been indicated in more detail only once and has 
to be added at corresponding places and to be adopted for the points labeled with $(\mathbf{a}, \mathbf{c})$ or $(\mathbf{c}, \mathbf{b})$ appropriately. In what follows, we only discuss the situation for $\mathbb{E}-$ Duom ((1)).
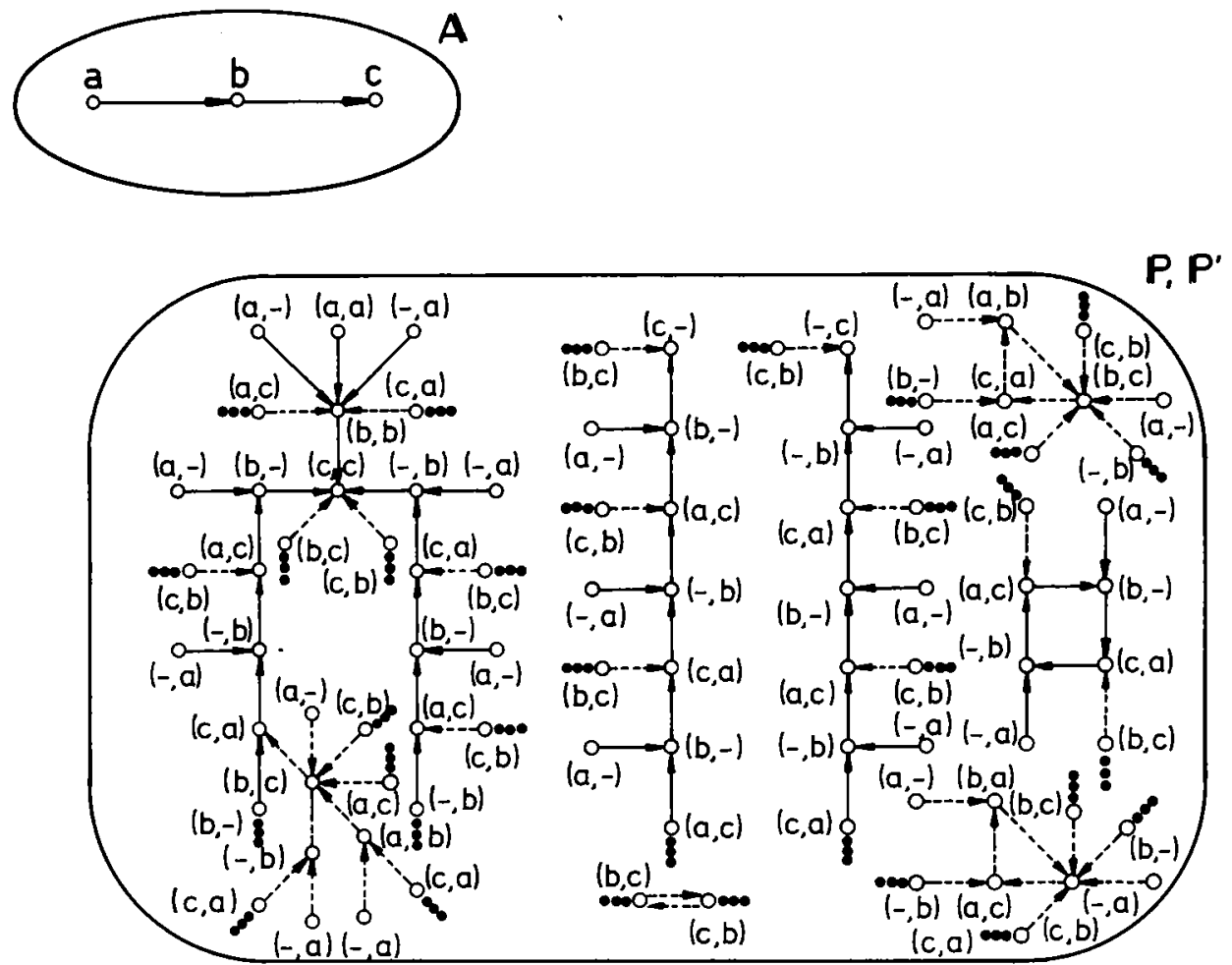

Figure 3: Fragments of the products $\mathbb{P}$ (in $(\mathbb{- g u o m}(1))$ ) and $P^{\prime}$ in con $f((1))$ ) of $A$ with $A$

It is easily realized that $p_{1}: \mathbb{P} \rightarrow \mathbb{A}$ and $p_{2}: \mathbb{P} \rightarrow \rightarrow \mathbb{A}$ are closed quomorphisms. And if, $\operatorname{say}\left(\mathbb{R},\left(\mathbf{r}_{1}: \mathbb{R}-\rightarrow \mathbb{A}, \mathbf{r}_{2}: \mathbb{R} \rightarrow \rightarrow \mathbb{A}\right)\right)$ is a product of $\mathbb{A}$ with $\mathbb{A}$ in $\mathbb{E}-\operatorname{Quom}((1))$, and if $\mathbf{p}: \mathbb{P}-\rightarrow \mathbb{R}$ is the closed quomorphism induced by $p_{1}$ and $p_{2}, i . e, p_{i}=r_{i}{ }^{\circ}$ for $i=1,2$, then one may realize that:

- $\mathbf{p}$ has to be defined on all of $\mathbf{P}$,

- if $p$ identifies any two elements $q$ and $q^{\prime}$ in $P$, then the labels $\left(p_{1}(q), p_{2}(q)\right)$ and $\left(p_{1}\left(q^{\prime}\right), p_{2}\left(q^{\prime}\right)\right)$ have to be the same and corresponding elements in the " $\varphi$-paths" starting with $q$ and $q^{\prime}$ respectively, also have to have the same labels. 
From the last property and Figure 3 one easily realizes that $p$ has to be injective, showing that a product of $A$ with $A$ in $(-$-Quom $((1))$ has to have $\mathbb{P}$ as a subalgebra - we even claim that it has to be isomorphic with $\mathbb{P}$, while in conf (1)) $\mathbb{P}^{\prime}$ can still be augmented by uncountably infinitely many connected components either ending in cycles with "sequences" for the labels of the form $\ldots \longrightarrow(b, c) \longrightarrow(c, b) \longrightarrow(b, c) \longrightarrow \ldots$ of different lengths alternating e.g. with "sequences" like $\ldots \longrightarrow(b, c) \longrightarrow(c, a) \longrightarrow(a, b) \longrightarrow(b, c) \rightarrow \ldots$ etc., or by components having no last element and no cycle and also containing sequences of different lengths labeled alternatingly by sequences of the above forms or similar ones.

These observations show "how monstrous" products may become in $E-\Omega u o m\left((1)_{\varphi \in \Omega}\right)$ and in $\operatorname{Conf}\left((1)_{\varphi \in \Omega}\right)$, and that for sources $\left(B,\left(f_{i}: B \longrightarrow \mathbb{A}_{i}\right)_{i \in I}\right)$ the "labels" $\left(f_{i}(b)\right){ }_{i \in I} \in \underset{i \in I}{X\left(A_{i} \cup\{-\}\right)}$ (with $-\bigcup_{i \in I} A_{i}$ and $f_{i}(b):=-$, if $f_{i}(b)$ is undefined) become of great importance.

since a terminal object represents all kinds of limits with an empty diagram - and hence an empty set of objects -, and since dually initial objects represent colimits of empty diagrams, we can restrict considerations in the following results to non-empty diagrams, i.e to non-empty index sets for the families of objects involved (terminal and initial objects for the considered categories are described in [4] in Theorems 1 and 1d, respectively).

Theorem 2 (Products). Let $\left(\mathbb{A}_{i}\right)_{i \in I}$ be any family of partial algebras of type $\tau$ to which the following constructions always refer $(I \neq \varnothing)$.

In $\operatorname{5om}(\tau)$ products always exist, and a typical representative $\left(\mathbb{A},\left(\mathbf{p r}_{i}: \mathbb{A} \longrightarrow \mathbb{A}_{i}\right)_{i \in I}\right)$, where we write $\mathbb{A}=: \Pi\left(\mathbb{A}_{i} \mid i \in I\right)$, is obtained in the following way:

Let $A$ be the cartesian product

$$
A:=X\left(A_{i} \mid i \in I\right):=\left\{a: I \longrightarrow U\left\{A_{i} \mid i \in I\right\} \quad \mid a(i) \in A_{i} \text { for all } i \in I\right\} ;
$$

For each $i \in I$ let $\mathbf{p r}_{\mathbf{i}}: \mathbf{A} \longrightarrow \mathbf{A}_{\mathbf{i}}$ be the $i-t h$ "natural projection", i.e. $\operatorname{pr}_{i}(\mathbf{a}):=\mathbf{a}(\mathbf{i})$ for all $\mathbf{a} \in \mathbf{A}$. 
For each $\varphi \in \Omega$ define

$\operatorname{dom} \varphi^{A}:=\left\{\underline{a \in A}{ }^{n} \varphi\right.$ for all $i \in I$ pr $\left.{ }_{i}^{\circ} \underline{a} \in \operatorname{dom} \varphi^{A^{A}}\right\} ;$

and if a belongs to $\operatorname{dom} \varphi^{A}$, then

$$
\varphi^{A}(\underline{a}):=\left(\varphi{ }^{A} i\left(p_{i}{ }^{\circ} \underline{a}\right) \mid i \in I\right) \text {. }
$$

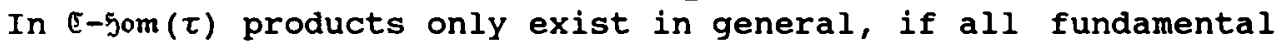
operations are unary, i.e. iff $\tau=(1), \varphi \in \Omega^{*}$

If the type is unary, then the typical product object is the subalgebra $B$ of the direct product object $A$ in the category $5 \mathrm{~cm}(\tau)$ - and the projections are the restrictions to $B$ of the projections $\mathbf{p r}_{i}$ - as defined above, where $B$ is the set of all sequences a of $A$ such that for each term $t$ in exactly one variable and the corresponding induced term operations $t_{i}^{A_{i}}$ one has:

for each $i$ and $j$ in $I, t^{\mathbb{A}} i(a(i))$ is defined iff $t^{A} j(a(j))$ is defined, i.e. all the components of a are term equivalent.

In Duom $(\tau)$ products do not exist in general, except for the trivial case that the set $\Omega$ of operation symbols is empty: $\Omega=\varnothing$. In this trivial case the typical product object $\mathbf{A}^{*}$ is

And for each $i \in I$

$$
\mathbf{A}^{*}:=U\left\{X\left\{\mathbf{A}_{j} \mid \mathbf{j} \in \mathbf{J}\right\} \mid \mathbf{J} \subseteq \mathbf{I}, \mathbf{J} \neq \boldsymbol{\sigma}\right\} \text {. }
$$

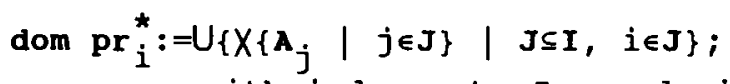

and if $a$ is a sequence with index set $J$, and if $i \in J$, then $\operatorname{pr}_{i}^{*}(a):=a(i)$.

In $(5-2 u o m(\tau)$ products exist only in general, if the similarity type $\tau$ either contains only constants or only unary operation symbols, i.e. iff either $\tau=(0)_{\varphi \in \Omega}$ or $\tau=(1)_{\varphi \in \Omega}$. If all $\varphi \in \Omega$ are nullary, then a typical product is set theoretically defined as in the case of sets with partial mappings (see above), and for each $\varphi \in \Omega$ one has:

for $\mathbf{J}_{\varphi}:=\left\{j \in I \mid \varphi^{\mathbb{A}} j\right.$ is defined in $\left.\mathbb{A}_{j}\right\}$ set $\varphi^{\mathbb{A}^{*}}:=\left(\varphi^{\mathbb{A}} j \mid j \in J_{\varphi}\right)$ if $\mathrm{J}_{\varphi} \neq \varnothing$, else - undefined.

The unary case is discussed together with the one for conformisms. 
In Conf $(\tau)$ product exists in general, iff $\tau=(1), \varphi \in \Omega^{0^{1}}$

Proof. The characterization of products in $50 m(\tau)$ is included in [2] (cf. 4.1.3 and 4.1.4). a

Let us consider $\tau-50 m(\tau)$. To begin with, we consider the case when $\tau$ specifies at least one nullary operation symbol, say $\varphi$. Let $A:=\{\mathbf{a}\}, B:=\{b\}$, and let $\varphi^{A}:=a$ be defined, while $\varphi^{\mathbb{B}}$ is undefined. Then there is no object $\mathbb{C}$ in $\mathbb{E}-50 m(\tau)$ allowing a closed homomorphism into $A$ (then $\varphi^{\mathbb{C}}$ would have to exist) as well as into $\mathbb{B}$ (then $\varphi^{\mathbb{C}}$ must not exist), hence there cannot exist a product of $A$ and $B$.

If $\tau$ specifies at least one at least binary operation symbol, then we consider the essentially binary situation the corresponding operation symbol will be $\varphi$ - sketched in Figure 3 (if an order of the arguments is not specified, we tacitly assume the parial operation to be commutative in that instance, i.e. that e.g. here $\left.\varphi^{\mathbb{B}}\left(b, b^{\prime}\right)=\varphi^{\mathbb{B}}\left(b^{\prime}, b\right)=b^{*}\right)$.

Suppose that $\mathbb{P}$ is a product object in $\mathbb{E}-5 \circ \mathrm{m}(\tau)$ for the family $(A, \mathbb{B})$. Since $\varphi^{\mathbb{A}}$ is total, $\varphi^{\mathbb{P}}$ has to be total, as can easily be shown. $\mathbb{P}$ has to have at least two elements, say $\mathbf{p}$ and $\mathbf{p}^{\prime}$, since there are two different closed homomorphisms $e$. g. from $A$ into $B$, whence the corresponding projection $\mathbf{p r}_{B}$ has to map $p$ onto $b$ and $\mathbf{p}^{\prime}$ onto $b^{\prime}$. Since $B$ is a proper partial algebra generated by $\left\{b^{\prime}, b^{\prime}\right\}, p_{B}$ has to be a surjective closed homomorphism and therefore $\varphi^{\mathbb{P}}$ has to be a proper partial operation, contradicting our first statement. Thus $\mathbb{E}-50 m(\tau)$ cannot have products in general, if $\tau$ specifies an at least binary operation.

If all fundamental operations are unary, then let us use the notation of the theorem. Lemma 5 in [4], tells us that the condition for a is necessary in order to be eligible as a member of $B$. Namely in a family $\left(f_{i}: \mathbb{C} \longrightarrow \mathbb{A}_{i} \mid i \in I\right)$ of closed

${ }^{1}$ since the corresponding construction is quite technical, we describe it only within the proof. The basic idea has already been described in [4] in connection with the construction of terminal objects in $\left[-5 a m\left((1)_{\varphi \in \Omega}\right)\right.$. Also compare the example preceding this theorem. 
homomorphisms each $f_{i}(c)$ is term equivalent with $c$. Hence, if $\mathbf{f}: \mathbb{C} \longrightarrow \Pi\left(A_{i} \mid i \in I\right)$ is the induced homomorphism in 5om $(\tau)$, then $f(c)$ will belong to $B$ for every element $c$ of $c$. Since $p r_{i} \circ f=f_{i}$ is closed for at least one (even all, and $I \neq \varnothing$ ) $i \in I$, $f$ is a closed homomorphism. It remains only to show that each $p_{i}:=p_{i} \mid B$ is a closed homomorphism from $B$ into $\mathbb{A}_{i}(i \in I)$. clearly, $p_{i}$ is a homomorphism. Assume $b \in B$ such that $\varphi^{A}{ }^{A}\left(p_{i}(b)\right)$ exists for some $i \in I$ and for some $\varphi \in \Omega$. Then, by the definition of $B, p_{i}(b)$ and each component of $b$ have the same directory (cf. [4], Definition 2), which contains $\varphi x$. Thus $\varphi^{\mathbb{A}}(b)$ exists for $A:=\Pi\left(\mathbb{A}_{i} \mid i \in I\right)$, and all components of $\varphi^{\mathbb{A}}(b)$ are term equivalent, too, as can easily be concluded in a similar way as it has been done in the proof of [4], Lemma 2 for the compatibility of $\theta_{c}$ with the fundamental operations. Therefore $\varphi^{A}(b) \in B$, showing that $p_{i}$ is a closed homomorphism. observe that the uniqueness of the induced morphism is guaranteed in the same way as in the larger category 5om $(\tau)$, which allows even more morphisms.

Let us now discuss the situation in $\$$ uom $(\tau)$. To begin with, let $\gamma \in \Omega$ be a nullary operation symbol. Let $A$ be $a$ oneelement partial algebra, in which $\gamma^{A}$ is defined, while all other operations are empty. We claim that the product of the family $(\mathbb{A}, \mathbb{A})$ does not exist in $\operatorname{guom}(\tau)$. Assume that $\mathbb{P}$ were such a product. Let us observe that $A$ allows exactly two quomorphisms into itself, the empty one $\varnothing$ and the identity id (cf. also Remark 1.(iii)). Thus, starting from $\mathbb{A}$ there are therefore four different pairs of quomorphisms into the pair $(A, A)$, out of which we consider $(i d, D)$ and $(i d, i d)$, and we denote by $\langle i d, \emptyset\rangle$ respectively $\langle i d, i d\rangle$ the induced quomorphisms from $A$ into $P$. Since in both cases the constant $\gamma^{\mathbb{A}}$ has to be mapped onto $\gamma^{\mathbb{P}}$, this yields a contradiction. Namely, <id,id> will have to map $\gamma^{A}$ onto $\left(\gamma^{A}, \gamma^{A}\right)$, while <id,ø> has to map it onto $\left(\gamma^{\mathbb{A}},-\right)$ ("-" designating an "empty component"). Thus $\gamma^{\mathbb{P}}$ cannot be defined uniquely.

Let us now assume, that $\tau(\varphi)=n>0$ for some $\varphi \in \Omega$. We consider an essentially unary situation with respect to $\varphi$. In 
what follows we shall treat $\varphi$ as if it were unary, since it will make no difference, if $n>1$. Moreover, let $A$ be a partial algebra with a carrier $A:=\{a, b\}, \varphi^{\mathbb{A}}:=\{(\mathbf{a}, \mathbf{b})\}$ and $\psi^{\mathbb{A}}:=\varnothing$ for all $\psi \in \Omega \backslash\{\varphi\}$. We claim that for the sequence $(A, A)$ there is no product in Duom $(\tau)$. Namely, assume that $\left(\mathbb{P},\left(\mathbf{p}_{1}, \mathbf{p}_{2}\right)\right)$ were a product in Duom $(\tau)$. As "test object" we also take $\mathbb{A}$, and we consider the following three sources starting from $A$ : $f:=(\{a \longmapsto a, b \longmapsto b\},\{a \longmapsto a\}), g:=(\{a \longmapsto a\},\{a \longmapsto a$, $\mathrm{b} \longmapsto \mathbf{b}\})$ and $\mathrm{h}:=(\{\mathbf{a} \longmapsto \mathbf{a}\},\{\mathbf{a} \longmapsto \mathbf{a}\})$ (where nothing is specified, the members of the families are undefined). In the pairs of $\mathbb{P}$ "I" will indicate that the corresponding component has to be undefined. Now, $\mathbf{f}$ implies that $((\mathbf{a}, \mathbf{a}),(\mathbf{b},-)) \in \varphi^{\mathbb{P}}$ while $\mathbf{g}$ implies that $((\mathbf{a}, \mathbf{a}),(-, b)) \in \varphi^{\mathbb{P}}$. Then either $\varphi^{\mathbb{P}}$ cannot be defined uniquely, or, if one wants to distinguish the two pairs $(a, a)$ above by some method (see the discussion of Eonf $(\tau))$, then the quomorphism induced by $h$ would not be unique. This shows that products cannot exist in general in Diom $(\tau)$, if there is at least one at least unary operation around.

Thus the only remaining case is, when $\Omega=\varnothing$. That $\left(\mathbf{A}^{*},\left(\mathbf{p r}_{\mathbf{i}}^{*} \mid i \in I\right)\right)$ is the product in the category of sets with partial mappings is also well known and easily realized. One should only observe that the empty sequence does not belong to $A^{*}$, since otherwise there could exist more than one induced partial mapping for a given source of partial mappings into the $A_{i} \cdot 0$

Let us now discuss the case of constants in $\mathcal{E}-$ Quom $(\tau)$. From Remark 1.(iv) it easily follows that for any similarity type $\tau$, if the product of any family $\left(\mathbb{A}_{i}\right) i \in I$ exists in

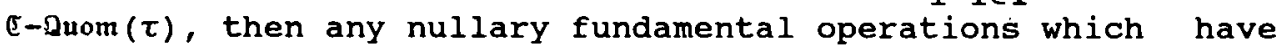
to exist in the product (induced by one of the components) will be defined as indicated in the theorem. And if $\tau$ possesses only nullary operation symbols then products will always exist that way. a

Before we get to the constructions for the unary similarity types in $(-2$ statements concerning $(-\operatorname{lnom}(\tau)$ and Eonf $(\tau)$. 
No products in $\mathfrak{C}$-Quom $((0,1))$ : Let $\tau$ have at least one nullary operation symbol, say $\varphi$, and at least one at least unary operation symbol, say $\psi$ - we shall assume $\psi$ to be unary in what follows -, and consider in $\mathbb{E - Q u o m}(\tau)$ two two-element partial algebras $A$ and $\mathbb{B}$, where $A=B:=\{\mathbf{a}, \mathbf{b}\}, \varphi^{\mathbb{A}}:=\mathbf{a}, \varphi^{\mathbb{B}}$ undefined, $\psi^{A}=\psi^{\mathbb{B}}:=\{\mathbf{a} \longmapsto \mathbf{b}\}$, and if there are further operations, they are empty both in $\mathbb{A}$ and $\mathbb{B}$. Moreover, consider the following two sources $f$ and $g$ of closed quomorphisms from $A$ into $(A, B)$ :

$f:=(\{a \longmapsto a, b \longmapsto b\},\{b \longmapsto b\}), g:=(\{a \longmapsto a, b \longmapsto b\}, d)$. If $\left(\mathbb{P},\left(\mathbf{p}_{1}: \mathbb{P}-\rightarrow \mathbb{A}, \mathbf{p}_{2}: \mathbb{P}-\rightarrow \mathbb{B}\right)\right)$ were a product of $\mathbb{A}$ and $B$ in (-Quom $(\tau)$, then $\varphi^{\mathbb{P}}$ would have to exist (see Remark 1.(iv)), labeled by $(\mathbf{a},-)$ (i.e. $\mathbf{p}_{1}\left(\varphi^{\mathbb{P}}\right)=\mathbf{a}, \mathbf{p}_{2}\left(\varphi^{\mathbb{P}}\right)$ undefined), and also $\psi^{\mathbb{P}}\left(\varphi^{\mathbb{P}}\right)$ would have to exist; however, because of $f$, its label would have to be $(a, b)$, while with respect to $g$ its label would have to be $(b,-)$, a contradiction. Thus in 5 -auom $((0,1))$ - and for "larger types" - products do not exist in general. a

No products in Eonf $((0))$ : Let $\tau$ specify a nullary operation symbol, say $\varphi$. Let $\mathbb{A}$ be a one-element partial algebra on $A:=\{\mathbf{a}\}$ with $\varphi^{\mathbb{A}}$ defined, and let $\mathbb{B}$ be a two-element discrete partial algebra on $B:=\left\{b, b^{\prime}\right\}$. Assume that $\left(\mathbb{P},\left(\mathbf{p}_{1}: \mathbb{P}-\rightarrow \mathbb{A}\right.\right.$, $\left.\left.\mathbf{p}_{2}: \mathbb{P}-\rightarrow \mathbb{B}\right)\right)$ is a product of $(A, B)$ in Conf $(\tau)$, and consider the following two sources $f$ and $g$ of conformisms from $A$ into $(A, B):$

$$
\mathbf{f}:=(\{\mathbf{a} \longmapsto \mathbf{a}\},\{\mathbf{a} \longmapsto \mathbf{b}\}), \mathbf{g}:=\left(\{\mathbf{a} \longmapsto \mathbf{a}\},\left\{\mathbf{a} \longmapsto \mathbf{b}^{\prime}\right\}\right) .
$$

$\varphi^{\mathbb{P}}$ has still to be defined, however with label $(\mathbf{a}, \mathbf{b})$ with respect to $f$ and with label $\left(\mathbf{a}, \mathbf{b}^{\prime}\right)$ with respect to $g$. Thus $\mathbf{p}_{2}\left(\varphi^{\mathbb{P}}\right)$ cannot be defined uniquely, and therefore the product does not exist. a 

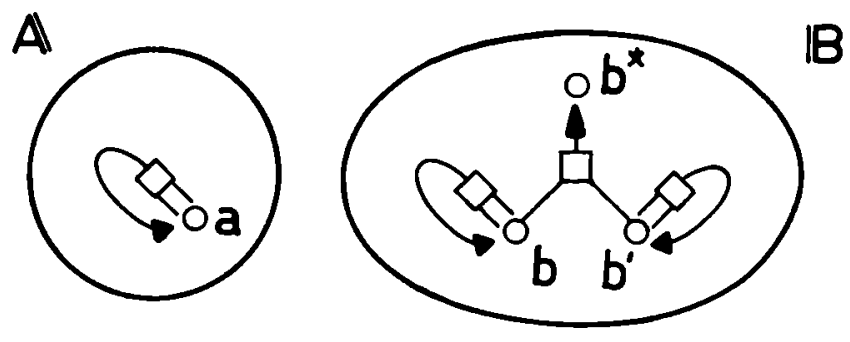

Figure 4: No products in $(5-50 m((2))$.

No products in $\mathrm{E}-\mathrm{guom}((2))$ and in Conf ((2)): In a similar way as for $5-50 m((2))$ in the proof of Theorem 1, [4], we give a cardinality argument in order to show that in certain cases a possible product. would have a proper class as carrier class. Assume that $\tau$ specifies an at least binary operation, say $\varphi$, and let us consider an essentially binary situation with respect to $\varphi$. It is well known (cf. [6], §9) that for any cardinality $n$ there exists a simple group, say $\mathbb{G}_{n^{\prime}}$ of that cardinality. Moreover, observe that, if $f: \mathbb{G} \longrightarrow \mathbb{B}$ is any groupoid homomorphism starting from a group $\mathbb{G}$, then $f$ is already a group homomorphism, and in particular, $f[G]$ is a subgroup of $B$, and the congruence relation on $G$ induced by $f$ is induced by a normal subgroup of $\mathbb{G}$. Thus, let $\mathbb{A}$ be a total, one-element groupoid (group!) and let $B$ be a discrete twoelement partial groupoid: $B:=\left\{b_{,} \mathbf{b}^{\prime}\right\}$. Assume that $\left(\mathbb{P},\left(\mathbf{p}_{1}: \mathbb{P}-\rightarrow \mathbb{A}, \mathbf{p}_{2}: \mathbb{P}-\rightarrow \mathbb{B}\right)\right)$ is a product of $(\mathbb{A}, \mathbb{B})$. For any infinite cardinality $n$ consider a source $\quad\left(f_{\mathbb{A}}: \mathbb{G}_{\mathfrak{n}} \longrightarrow \mathbb{A}\right.$, $\left.f_{B}: \mathbb{G}_{n} \longrightarrow \mathbb{B}\right)$ of closed quomorphisms/conformisms, where $f_{A}$ is a group homomorphism and $f_{B}$ is for (Eonf((2)) an arbitrary surjective mapping, while for $\mathbb{E}-\mathfrak{D u m}((2)) \mathbf{f}_{\mathbb{B}}$ is defined on exactly two permutations, say $(x \mathbf{y})$ and $\left(x^{\prime} y^{\prime} z^{\prime}\right)$ (in the notation as cycles) for disjoint 3 -element subsets. $\{x, y, z\}$ and $\left\{x^{\prime}, y^{\prime}, z^{\prime}\right\}$ of $\mathbb{G}_{\mathfrak{n}^{\prime}}$ and it maps $(x \mathbf{y})$ to $b$ and ( $x^{\prime} y^{\prime}$ $\left.z^{\prime}\right)$ to $b^{\prime}$. Then $\left\{(\mathbf{x} \mathbf{y} z),\left(\mathbf{x}^{\prime} \mathbf{y}^{\prime} \mathbf{z}^{\prime}\right)\right\}$ is the carrier of a 
discrete relative subalgebra of $\mathbb{G}_{n}$ and $\boldsymbol{f}_{B}$ is indeed a closed and surjective quomorphism. Since $A$ is total, the preimage of A with respect to $\mathbf{p}_{1}$ is a total subalgebra, say $\mathbb{P}^{\prime}$, of $\mathbb{P}$. And the closed quomorphism/conformism $f: \mathbb{G}_{n}-\rightarrow \mathbb{P}$ induced by $\left(f_{A}, f_{B}\right)$ has to be totally defined, and to $\operatorname{map} G_{n}$ into $P^{\prime}$ therefore it has to be a group homomorphism. since $f_{B}=p_{2} \circ f$ is not constant, the kernel of $f$ (normal subgroup) cannot be all of $G_{n}$. Thus $f$ has to be injective, since $\mathbb{G}_{n}$ is simple. This implies that for any infinite group $G_{n}$ there has to exist an injective mapping of $G_{n}$ into $P$, but is impossible, since $P$ has to be a set and therefore has some cardinality itself. This shows that in (5-Ouom((2)) and in Conf((2)) there cannot be products in general (whose carriers are sets!). a

Products in $\left(\tau-\Omega\right.$ uom $(\tau)$ and in $\operatorname{conf}(\tau)$ for $\tau=(1)_{\varphi \in \Omega}$ : If $\tau=(1)_{\varphi \in \Omega}$ in the case of closed quomorphisms or conformisms, then the product of $\left(A_{i}\right)_{i \in I}$ is constructed as follows (we

2 The idea for the relatively technical description below is the following one, which is similar to - however still more complicated than - the one for the description of terminal objects in $[-50 m(\tau)$ for a unary similarity type. one has to guarantee that the start object $B$ of every source $\left(B,\left(f_{1}: B-\rightarrow\right.\right.$ $\left.A_{1}\right)_{i \in I}$ ) of closed quomorphisms respectively conformisms allows exactly one quomorphism/conformism into the product object such that it is "compatible" with the source of projections, which have to be closed quomorphisms/conformisms. However we only need "minimal" start objects, i.e. in a given source we may restrict the start object $B$ to the elements which are in the domain of at least one $f_{i}$, and the structure to what is really needed in order that all $f_{1}$ remain closed quomorphisms/conformisms, and we assume that $B$ is connected we shall call sources with such a minimal start object minimal sources. We take the disjoint union $U=\left(U, \quad\left(\varphi^{U}\right)_{\varphi \in \Omega}\right)$ of a representative system of certain start objects for nonisomorphic minimal sources, where we restrict considerations to such isomorphism types of minimal sources, whose start objects do not allow any closed non-injective homomorphism compatible with the sources onto any other start object of any other minimal source. Then we can prove that $U$ is really a set, and that we get at least one closed quomorphism/conformism from the start object into $U$. We have to factor $U$ through an appropriate closed congruence relation - closed homomorphisms are closed quomorphisms/conformisms - 
shall speak of morphisms in order to refer to closed quomorphisms and conformisms, respectively, for the definition of minimal source see footnote 2 ).

Let $X^{\prime}:=\left\{D:=\left(\mathbb{D},\left(f_{i}: D-\rightarrow A_{i}\right)_{i \in I}\right) \mid \mathcal{D}\right.$ is a minimal source of morphisms\}, let $\Omega^{\star}:=\Omega \dot{u}^{*}$ and let $\tau^{\star}$ be the corresponding similarity type, where $\tau^{*}\left(a^{*}\right)=1$ for each $a \in \lambda^{*}$ and $\tau^{*}(\varphi)=\tau(\varphi)$ for each $\varphi \in \Omega$. And for $D:=\left(\mathbb{D},\left(f_{i}: \mathbb{D}-\rightarrow \mathbb{A}_{i}\right)_{i \in I}\right) \in R^{\prime}$ let $\mathbb{D}_{D}:=$ $=\left(D,\left(\varphi^{\mathbb{D}}\right)_{\varphi \in \Omega} \cup\left(a^{\star D}\right) a \in \lambda^{*}\right)$, where, for each $a \in A^{*}$ :

$\operatorname{dom} a^{* D}:=\left\{d \in D \mid\left(f_{i}(d) \mid i \in I\right.\right.$ and $\left.\left.d \in \operatorname{dom} f_{i}\right)=a\right\}$, and if $d \in \operatorname{dom} a^{\star D}$, then $a^{\star D}(d):=d$. Thus $d \in D$ will belong to the domain of $a^{*}$ for exactly one $\mathbf{a} \in \boldsymbol{A}^{*}$, which is called the label $\mathrm{I}(\mathrm{d})$ of $\mathbf{d}$. Two sources $D:=\left(\mathbb{D},\left(f_{i}: \mathbb{D}-\rightarrow \mathbb{A}_{i}\right)_{i \in I}\right)$ and $\mathbb{E}:=\left(\mathbb{E},\left(g_{i}: \mathbb{E}-\rightarrow \mathbb{A}_{i}\right)_{i \in I}\right)$ in ' will be said to be isomorphic if there is an isomorphism $j: \mathbb{D} \longrightarrow \mathbb{E}$ such that for each $i \in I$ one has $g_{i}{ }^{\circ}=f_{i}$.

Then let $R$ be a representative system of all isomorphism types of sources in $\boldsymbol{R}^{\prime}$ such that the partial algebra $\mathbb{D}_{\mathfrak{D}}$ corresponding to its start object $\mathbb{D}$ is closed-simple. Let $U$ be the disjoint union (coproduct in $50 m\left(\tau^{*}\right)$ ) of all $\mathbb{D}_{\mathfrak{D}}$ with $\mathfrak{D \in R}$. Finally, let $\theta^{*}$ be the largest closed congruence relation on $U$. Let $\mathbb{P}^{\star}:=\mathbb{U} / \theta^{*}$, and let $\mathbb{P}$ be the $\tau$-reduct of $\mathbb{P}^{*}$. Moreover, for each $i \in I$ let the $i-t h$ projection $p_{i}: \mathbb{P} \rightarrow \mathbb{A}_{i}$ be $\operatorname{defined~-~}$ with respect to the labelling $l$ in $\mathbb{P}^{*}$ - as follows: for each $q \in P$ let $q \in \operatorname{dom} p_{i}: \Leftrightarrow \quad i \in \operatorname{dom} I(q)$, and if $q \in \operatorname{dom} p_{i}$, then $p_{i}(q):=l(q)(i)$. We claim that $\left(\mathbb{P},\left(p_{i}: \mathbb{P}-\rightarrow \mathbb{A}_{i}\right)_{i \in I}\right)$ is a product of $\left(A_{i}\right)_{i \in I}$ in $(\tau-\operatorname{aum}(\tau)$ respectively in $\operatorname{Conf}(\tau)$ for $a$ multi-unary type $\tau$.

Let us prove this claim. It is easily seen that from the

respecting the compatibility with the closed suurce quomorphisms/ conformisms. In order to encode (minimal) sources algebraically, we inţroduce new unary partial operations $\mathbf{a}$, where $a \in \mathbf{A}^{*}$. And $\mathbf{a}$ is defined on $b \in \mathbf{B}-$ and then takes the value $b-$, iff for $a l l \quad l \in I$ a(1) $=f_{1}$ (b) (if one side is defined, then so has to be the other side). This will allow us to restrict considerations to minimal closed-simple partial $\tau$-algebras instead of considering sources with the required properties. 
$\tau^{\star}$-extensions $\mathbb{D}_{\mathfrak{D}}$ of the start objects of (minimal) sources $\mathfrak{D}:=\left(\mathbb{D},\left(\mathbf{p}_{\mathbf{i}}: \mathbb{D}-\rightarrow \mathbb{A}_{\mathbf{i}}\right)_{i \in I}\right)$ the source itself can be totally recovered - note that for an arbitrary source $D$, if an element of $D$ is in the domain of no $f_{i}$, then it has no label, i.e. for no $a \in \mathbf{A}^{*} \mathbf{a}^{*}$ is defined on it. And when we consider a closed congruence relation $\theta$ of $\mathbb{D}_{\mathfrak{D}}$, then [4], Lemma 6 tells us that with respect to the closed quotient homomorphism nat $\theta: \mathbb{D} \longrightarrow$ $\mathbb{D} / \theta$ there exists a source $D^{\star}:=\left(\mathbb{D} / \theta,\left(g_{i}: \mathbb{D} / \theta-\rightarrow \mathbb{A}_{i}\right)_{i \in I}\right)$ such that, for each $i \in I, g_{i}{ }^{\circ n^{\prime} t_{\theta}}=\mathbf{f}_{i}$. If $\mathbf{f : \mathbb { D }} \rightarrow \mathbb{P}$ and $\mathbf{g}: \mathbb{D} / \theta-\rightarrow \mathbb{P}$ are the morphisms into the product object of the family $\left(\mathbb{A}_{i}\right)_{i \in I}$, it is easy to realize that $f=g \circ$ nat ${ }_{\theta}$. This argument shows that in order to get an idea, how a possible product of a given family of partial algebras will look like, we can restrict considerations, without loss of generality, to sources $\mathfrak{D}$, for which $\mathbb{D}_{\mathfrak{D}}$ is closed-simple.

It is similarly obvious that for the construction of $\mathbb{P}$ one can restrict considerations to what we call minimal sources. Then we can argue as in the case of the existence of a terminal object in (5-5om( $\tau$ ) for a unary similarity type (see [4]) that there is up to isomorphism only a set $\pi$ of connected closed-simple $\tau^{\star}$-algebras (cf. [4]). And the coproduct, say $\left(\left(j_{\mathfrak{D}}: \mathbb{D}_{\mathfrak{D}}-\rightarrow \mathbb{U}\right)_{\mathfrak{D} \in \mathfrak{R}^{\prime}} \mathbb{U}\right)$, in $\mathfrak{J o m}^{\star}\left(\tau^{\star}\right)$ of this set exists, and $\mathbb{U}$ is the disjoint union of the $\mathbb{D}_{\mathfrak{D}}$ in $x$ (see [2], Proposition 4.3.5). If $\theta^{*}$ designates the largest closed $\left(\tau^{*}-\right)$ congruence on $\mathbb{U}$, and if $\mathbb{P}^{*}:=\mathbb{U} / \theta^{*}$, then it is easy to realize that each partial $\tau^{*}$-algebra $\mathbb{D}_{\mathfrak{D}}$ stemming from a source $\mathbb{D}$ into $\left(\mathbb{A}_{i}\right)_{i \in \mathbf{E}} \mathbf{I}$ allows at least one closed homomorphism/conformism into $\mathbb{P}^{\mathbf{P}}$, since each connected component of $\mathbb{D}_{\mathfrak{D}}$ allows one according to our construction.

Next we want to show in the case of $(\mathbb{T}$-Duom $(\tau)$ (Eonf $(\tau)$ ) that all projections are closed quomorphisms respectively conformisms:

First let $\mathbf{q}, \mathbf{q}^{\prime} \in \mathbf{P}$ - with labels $\mathfrak{l}(\mathbf{q})=\mathbf{a}, \mathfrak{l}\left(\mathbf{q}^{\prime}\right)=\mathbf{a}^{\prime}$ - be such that $\varphi^{\mathbb{P}}(q)=q^{\prime}$. Then, by the definition of $\mathbb{P}$, there exist $D \in \mathbb{R}$, say $\mathfrak{D}=\left(\mathbb{D},\left(f_{i}: \mathbb{D}-\rightarrow \mathbb{A}_{i}\right)_{i \in I}\right)$ with the induced closed morphism $f: \mathbb{D}-\rightarrow \mathbb{P}$, and $d, d^{\prime} \in \mathbb{D}$ with $\mathbf{f}(\mathbf{d})=\mathbf{q}$ and $\mathbf{f}\left(\mathbf{d}^{\prime}\right)=\mathbf{q}^{\prime}$ (observe that also $I(d)=\mathbf{a}$ and $\left.l\left(d^{\prime}\right)=\mathbf{a}^{\prime}\right)$ such that $\varphi^{\mathbb{D}}(\mathbf{d})=\mathbf{d}^{\prime}$. 
Let us first consider the case of closed quomorphisms: since each $f_{j}$ is a quomorphism, we have for each $i \in I$ satisfying $d, d^{\prime} \in \operatorname{dom} f_{i}$ that $\varphi^{\mathbb{A}}{ }^{1}\left(p_{i}(q)\right)=\varphi^{\mathbb{A}}{ }^{1}\left(f_{i}(d)\right)=f_{i}\left(d^{\prime}\right)=p_{i}\left(q^{\prime}\right), i . e$. this holds for all indices for which $q \in$ dom $p_{i}$ as well as $q^{\prime} \in \operatorname{dom} p_{i}$. Thus, for $\varepsilon-\operatorname{Duom}(\tau)$, each $p_{i}$ is a quomorphism.

Moreover, now including the case of conformisms, let $q \in P$, and let us assume that for some $i \in \operatorname{dom} I(q)-\varphi^{A}{ }^{A}\left(p_{i}(q)\right)$ exists. Let $D=\left(\mathbb{D},\left(f_{i}: \mathbb{D}-\rightarrow \mathbb{A}_{i}\right)_{i \in I}\right) \in \mathbb{R}$ be a minimal source of closed quomorphisms respectively of conformisms containing deD such that $f_{i}(d)=p_{i}(q)$, and such that, for the induced "canonical" morphism $f: \mathbb{D}-\rightarrow \mathbb{P}$, one has $f(d)=q$. Such a source has to exist! since $f_{i}$ is a closed quomorphism respectively a conformism, $\varphi^{\mathbb{D}}(\mathrm{d})$ exists since $\varphi^{\mathbb{A}_{i}}\left(\mathbf{p}_{i}(q)\right)=\varphi^{\mathbb{A}}\left(f_{i}(d)\right)$ exists. Since $D \in \mathbb{R}$, the construction of $\mathbb{P}$ implies that then also $\varphi^{\mathbb{P}}(q)$ exists, showing that $p_{i}$ is a closed quomorphism respectively a conformism and this is true for all $i \in I$.

Finally let us prove the uniqueness of an induced morphism into $\mathbb{P}$ : Let therefore $D=\left(\mathbb{D},\left(f_{i}: \mathbb{D}-\rightarrow \mathbb{A}_{i}\right)_{i \in I}\right)$ be any source, and assume that $f, g: \mathbb{D} \rightarrow \mathbb{P}$ are two induced morphisms such that $\mathbf{p}_{\mathbf{i}} \circ \mathbf{f}=\mathbf{p}_{\mathbf{i}} \circ \mathbf{g}=\mathbf{f}_{i}$ for each $i \in I$. Because of the commutativity requirements $f$ and $g$ have to have the same domain, say $D^{\prime} \subseteq D$.

In the case of closed quomorphisms $f$ as well as $g$ are closed homomorphisms from a relative $\left(\tau^{*}-\right)$ subalgebra $\mathbb{D}^{*}$ of $\mathbb{D}_{\mathfrak{D}}$ into $\mathbb{P}^{\star}$, and since $\mathbb{P}^{\star}$ is closed-simple, [4], Lemma 1 implies that $\mathbf{f}=\mathbf{g}$.

In the case of conformisms let $\mathbb{D}_{f}$ be the weakest relative subalgebra of $\mathbb{D}_{\mathfrak{D}}$ allowing all $f_{i}$ to be conformisms. Without loss of generality we may assume that $f \mid \mathbb{D}_{\mathbf{f}}: \mathbb{D}_{\mathbf{f}} \rightarrow \mathbb{P}^{*}$ is the closed $\tau^{\star}$-homomorphism connected with the construction of $\mathbb{P}^{*} .^{3}$

${ }^{3}$ This means that each connected component of $\mathbb{D}_{\mathfrak{D}}^{\prime}$ is factorized by its largest closed congruence relation; then each of these quotient mappings is followed by the embedding into $v$, which is unique, since each domain is closed-simple; and the disjoint union (coproduct) of all these closed homomorphisms is followed by nat ${ }_{\theta}^{*}$. 
Moreover, let $\mathbb{D}_{g}$ be the weakest relative subalgebra of $D_{D}$ such that $g \mid D_{g}: \mathbb{D}_{g}-\rightarrow \mathbb{P}^{*}$ as well as all $f_{i}$ are conformisms. Observe that $D_{f}=D_{g}=: D^{\prime}$ - again because of the commutativity requirements. Moreover, the commutativity requirements and the assumptions on $\mathbf{g}$ also imply that $\mathbb{D}_{\mathbf{g}}$ is at least a weak relative subalgebra of $\mathbb{D}_{f}$. Namely assume that $\varphi^{g}(d)=d^{\prime}$. Because of the minimality requirements on $\mathbb{D}_{g}$ this either implies $g(d) \in \operatorname{dom} \varphi^{\mathbb{P}}$ or $f_{i}(d) \in \operatorname{dom} \varphi^{A_{i}}$. In the latter case we immediately get $d \in \operatorname{dom} \varphi^{\mathbb{D}_{f}}$. In the former case the definition of $P^{*}$ implies the existence of some $i \in I$ such that $p_{i} \circ g(d) \in$ dom $\varphi{ }^{A_{i}}$. Therefore dedom $\varphi^{D_{f}}$, showing in both cases that $\mathbb{D}_{\mathbf{g}} \mathbb{D}_{\mathbf{f}}$. By symmetry we therefore get $\mathbb{D}_{\mathbf{g}}=\mathbb{D}_{\mathbf{f}}$. Therefore $f \mid D_{f}$ and $g / D_{f}$ are closed homomorphisms from $D_{f}$ into $P^{*}$, and the same argument as for closed quomorphisms now shows $f=g$. This finishes the proof in the case of closed quomorphisms and conformisms for multi-unary partial algebras and therefore also the one of Theorem 2 .

Theorem 2d (Coproducts). Let $\left(\mathbb{A}_{i}\right)_{i \in I}$ be any family of partial algebras of type $\tau$ to which the following constructions always refer $(I \neq \varnothing)$.

In Som $(\tau)$ coproducts always exist, and a typical representative $\left(\left(c_{i}: A_{i} \longrightarrow A\right)_{i \in I}, A\right)$ is obtained in the following way, if $\Omega^{(0)}=\varnothing$ : Let $A$ be the "disjoint union":

$$
\left.\mathbf{A}:=U_{\left\{\mathbf{A}_{i} \times\{i\} \mid\right.} \mid i \in I\right\}:=\left\{(\mathbf{a}, \mathbf{i}) \mid \mathbf{a} \in \mathbf{A}_{\mathbf{i}}, i \in I\right\}=: \dot{U}\left(\mathbf{A}_{i} \mid i \in I\right) .
$$

And the structure of $A=: U\left(A_{i} \mid i \in I\right)$ is obtained as the "disjoint union" of the structures of the $\mathbb{A}_{i}(i \in I), i . e$. for each $\varphi \in \Omega$ we have:

$$
\operatorname{dom} \varphi^{\mathbb{A}}:=\left\{\left(\left(a_{1}, i\right), \ldots,\left(a_{n_{\varphi}}, i\right)\right) \mid i \in I \text { and }\left(a_{1}, \ldots, a_{n_{\varphi}}\right) \in \operatorname{dom} \varphi^{\mathbb{A}_{i}}\right\},
$$

and if $\left(\left(a_{1}, i\right), \ldots,\left(a_{n_{\varphi}}, i\right)\right) \in \operatorname{dom} \varphi^{A}$ for some $i \in I$, then

$$
\varphi^{\mathbb{A}}\left(\left(a_{1}, i\right), \ldots,\left(a_{n_{\varphi}}, i\right)\right):=\left(\varphi^{A}\left(a_{1}, \ldots, a_{n_{\varphi}}\right), i\right) .
$$

For each $i \in I$ the corresponding natural injection $\iota_{i}: \mathbb{A}_{i} \rightarrow \mathbb{A}$ is
defined by 


\section{$\iota_{i}(\mathbf{a}):=(\mathbf{a}, \mathbf{i})$ for each $\mathbf{a} \in \mathbf{A}_{i}$.}

If $\Omega^{(0)} \neq \sigma$, then first construct $A^{\prime}$ like $A$ above with respect to $\Omega^{\prime}:=\Omega \backslash \Omega(0)$, then take the quotient of $A^{\prime}$ with respect to the congruence relation $R^{\prime}$ of $A^{\prime 4}$ generated by the following relation $\mathbf{R}^{\prime \prime}$ :

$R^{\prime \prime}:=\left\{\left(\left(\varphi^{A}, i\right),\left(\varphi^{A} j, j\right)\right) \mid \varphi \in \Omega(0)\right.$ is defined in $A_{i}$ as well as in $\left.A_{j}\right\}$;

then finally define on $\mathbf{A}:=\mathbf{A}^{\prime} / \mathbf{R}^{\prime}$ for $\varphi \in \Omega \backslash \Omega^{(0)}$ the structure $\varphi^{\mathbb{A}}$ to be the quotient structure $\varphi^{A^{\prime} / R^{\prime}}$; and for $\varphi \in \Omega^{(0)}$ define $\varphi^{A}$ to be the congruence class containing all $\left(\varphi^{\mathbb{A}_{i}}, i\right)$, if some $\varphi^{\mathbb{A}_{i}}$ is defined for some $i \in I$, and let $\varphi^{A}$ be undefined, else.

In $\tau-50 m(\tau)$ and in $\operatorname{conf}(\tau)$ a coproduct only exists in general, if $\tau=(1)_{\varphi \in \Omega}$, i.e. if $\tau$ is unary, and in auom $(\tau)$ a coproduct exists in general only if $\Omega^{(0)}=\varnothing$. In $\tau$-2uom $(\tau)$ coproducts only exist in general if the similarity type is either unary $-\tau=(1)_{\varphi \in \Omega}$ - or only nullary $-\tau=(0)_{\varphi \in \Omega}$. And in all the cases without constants $\left(\left(\iota_{i}: \mathbb{A}_{i} \longrightarrow \|\left(\mathbb{A}_{i} \mid i \in I\right)\right)_{i \in I}, U\left(\mathbb{A}_{i} \mid i \in I\right)\right)$ is defined as for 5om $(\tau)^{5}$.

In $\left(\mathbb{-}\right.$-Duom $\left((0)_{\varphi \in \Omega}\right)$ one may define a coproduct $\left(\left(\iota_{i}: \mathbb{A}_{i}-\rightarrow \mathbb{C}\right)_{\left.i \in I^{\mathbb{C}}\right) \text { as follows (observe } \Omega=\Omega(0)}\right)$ : set $\Omega^{\star}:=\{\varphi \in \Omega \mid$ $\varphi^{A} i$ exists for every $\left.i \in I\right\}$, and let $\theta$ be the equivalence relation on $\Omega$ generated by the relation

$\mathbf{R}:=\left\{(\varphi, \psi) \mid \varphi, \psi \in \Omega\right.$, and there exists $i \in I$ such that $\left.\varphi^{\mathbb{A}} i_{=\psi}{ }^{\mathbb{A}} i\right\} ;$ then define

$$
\mathrm{C}:=\dot{U}\left\{\mathbf{A}_{i} \backslash\left\{\varphi^{\mathbb{A}} \mathbf{i} \mid \varphi \in \Omega\right\} \quad \mid \quad i \in \mathbf{I}\right\} \dot{\cup}\left\{[\varphi] \dot{\theta} \mid \varphi \in \Omega,[\varphi] \theta \subseteq \Omega^{\star}\right\}^{6},
$$

and let, for $\varphi \in \Omega, \varphi^{\mathbb{C}}:=[\varphi] \theta$, if $[\varphi] \theta \subseteq \Omega^{*}$, else undefined. The -

${ }^{4}$ The corresponding natural projection will be denoted by $\operatorname{nat}_{R^{\prime}}: A^{\prime} \longrightarrow A^{\prime} / R^{\prime}$.

${ }^{5}$ observe that here $\Omega^{(0)}=\varnothing$.

${ }^{6}[\varphi] \theta$ designates the equivalence class of $\varphi$ with respect to the equivalence relation $\theta$. 
not necessary injective - "injections" $\iota_{i}$ (ifI) are defined for $a \in \lambda_{i}$ by

$\iota_{i}(a):=\left\{\begin{array}{l}(a, i), \text { if } a \in \lambda_{i} \backslash\left\{\varphi^{A} \mid \varphi \in \Omega\right\}, \\ {[\varphi] \theta, \text { if } a=\varphi{ }_{i}, \varphi \in \Omega, \text { and }[\varphi] \theta \subseteq \Omega^{*},} \\ \text { undefined, else. }\end{array}\right.$

Proof. For $\operatorname{som}(\tau)$ the description of the coproduct is discussed in [2], Construction 4.3.4 and Proposition 4.3.5. That the same construction of taking the disjoint union of the partial algebras under consideration - no constants! - also works in the cases $E-50 m\left((1)_{\varphi \in \Omega}\right), \quad E-\operatorname{auom}\left((1)_{\varphi \in \Omega}\right)$ and Conf $\left((1)_{\varphi \in \Omega}\right)$ as well as for $\operatorname{Dum}(\tau)$ with $\tau(\varphi) \geq 1$ for each $\varphi \in \Omega$ is easily seen. $\square$

No coproducts in $\mathbb{E}$-Duom $((0,1))$ :

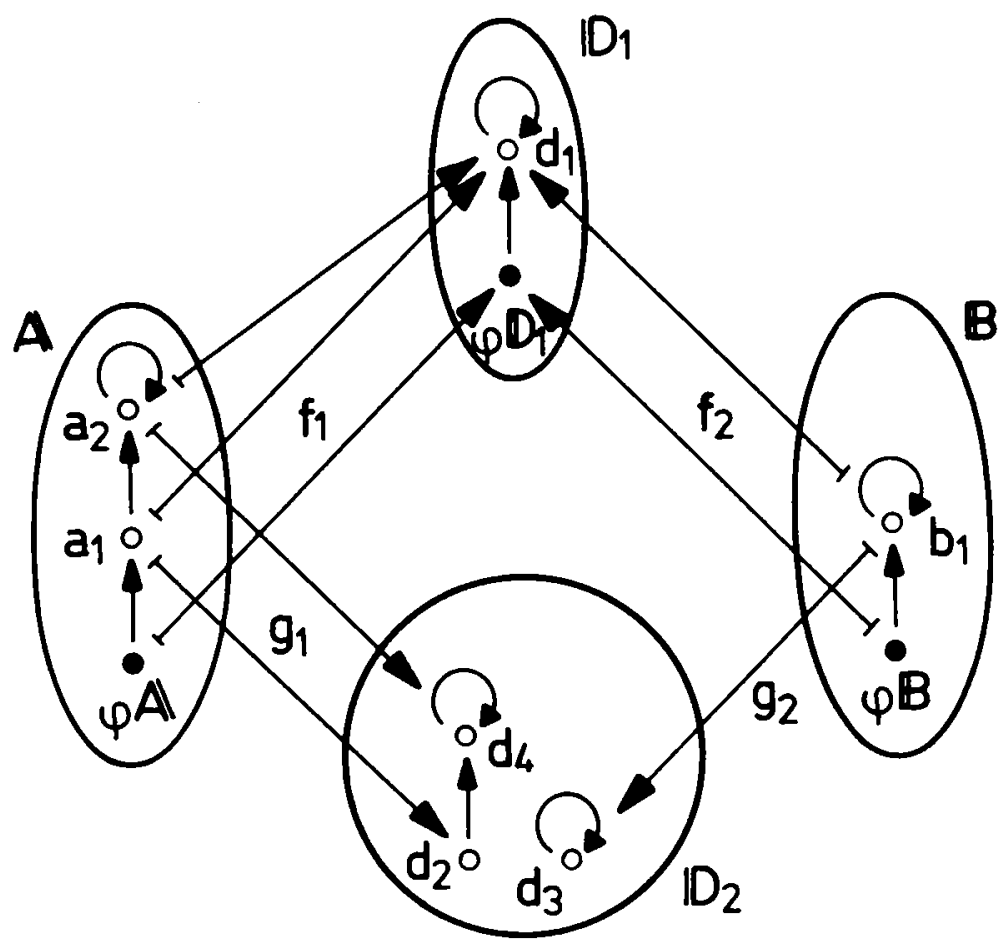

Figure 5: No coproduct in general in $E$-Duom $((0,1))$. 
We claim that the coproduct in $\mathbb{E - D u m}((0,1))$ and "corresponding" categories of the partial algebras $A$ and $B$ as depicted in Figure 5 does not exist: Consider the two sinks $\left(\left(f_{1}, f_{2}\right), D_{1}\right)$ and $\left(\left(g_{1}, g_{2}\right), D_{2}\right)$ as shown in Figure 5 . If $\left(\left(\iota_{1}, \iota_{2}\right), C\right)$ were a coproduct of $A$ and $B$ in $(E$-quom $((0,1))$, it would have to look like $\left(\left(f_{1}, f_{2}\right), D_{1}\right)$, since $\varphi^{\mathbb{C}}$ would have to be defined (see Remark 1.(iv)). However, it would not allow an induced closed quomorphism into $\mathbb{D}_{2} \cdot \square$

No coproducts in $\mathbb{E}-\operatorname{Duom}((0))$, Conf $((0))$, and Duom $(0))$ : Let $\tau$ specify a nullary partial operation, say $\varphi$, let $A$ and $B$ be any partial algebras of type $\tau$ such that $\varphi^{\mathbb{A}}$ is defined and $\psi^{B}$ is undefined. Then there is no partial algebra $\mathbb{C}$ into which $\mathbb{A}$ as well as $B$ allow a closed homomorphism.

In order to show that there cannot be coproducts in general in $\operatorname{conf}(\tau)$, when there are fundamental constants around, consider the situation sketched in Figure 6 , where $h_{1}$ and $g_{2}$ designate empty conformisms:

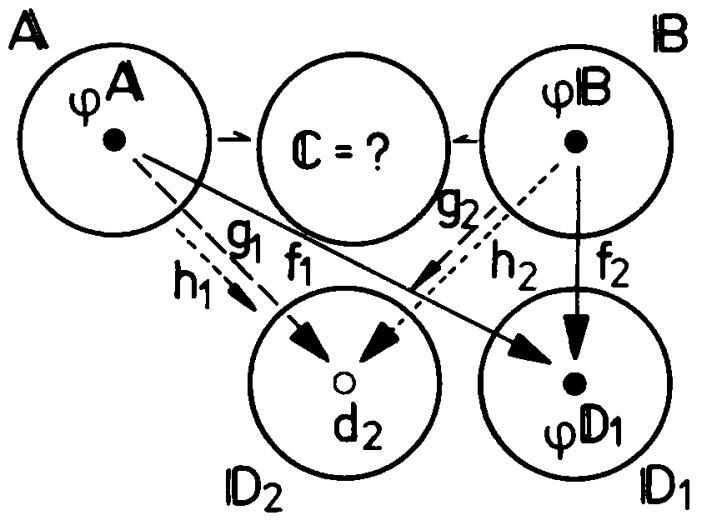

Figure 6: There are no coproducts in general in $\operatorname{conf}((0))$.

There is no coproduct of $A$ and $B$ - we would have called it c - in Conf $(\tau)$, where $\tau$ specifies a nullary operation symbol, since the "injections" from $A$ and $B$ into $\mathbb{C}$ would have to map the constants $\varphi^{A}$ and $\varphi^{\mathbb{B}}$ (with respect to the sink 
$\left.\left(\left(f_{1}, f_{2}\right), \mathbb{D}_{1}\right)\right)$ onto a constant $\varphi^{\mathbb{C}}$, while the sinks $\left(\left(g_{1}, g_{2}\right), \mathbb{D}_{2}\right)$ respectively $\left(\left(h_{1}, h_{2}\right), D_{2}\right)$ of conformism require different images of $\varphi^{\mathbb{A}}$ and $\varphi^{\mathbb{B}}$.

Finally, let us realize that $f_{1}$ and $f_{2}$ in Figure 6 are also quomorphisms, showing that the embeddings of $\mathbb{A}$ and $B$ into a possible coproduct $\mathbb{C}$ in suom $(\tau)$ would have to map the constants $\varphi^{\mathbb{A}}$ and $\varphi^{\mathbb{B}}$ onto $\varphi^{\mathbb{C}}$. However, if we replace, say $\mathbb{f}_{1}$, by the empty closed quomorphism into $\mathbb{D}_{1}$, then the corresponding "injection" must not map $\varphi^{\mathbb{A}}$ onto $\varphi^{\mathbb{C}}$, a contradiction. a

No coproducts in $\mathbb{E}-5 \circ m((2)), \mathbb{E}-\operatorname{arom}((2))$ and $\operatorname{Conf}((2))$ :

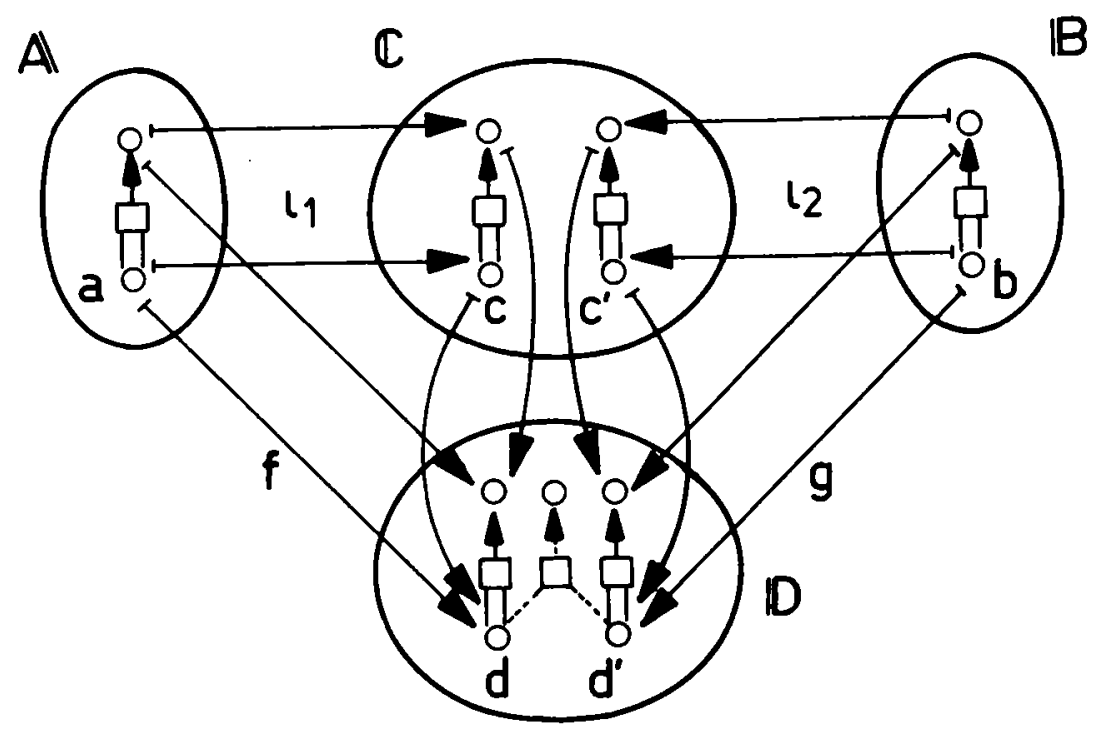

Figure 7: Coproducts do not exist in general in $\mathbb{C}-50 \mathrm{~m}((2))$

Let $\tau$ specify an at least binary operation symbol, say $\varphi$, and let $A, B$ and $\mathbb{D}$ be three partial algebras as depicted in Figure 7 together with closed homomorphisms $f: \mathbb{A} \longrightarrow \mathbb{D}$ and $\mathbf{g}: \mathbb{B} \longrightarrow \mathbb{D}$. Assume that $\left(\left(\iota_{1}, \iota_{2}\right), \mathbb{C}\right)$ is a coproduct of $\mathbb{A}$ and $\mathbb{B}$ 
in any of the three categories. Then $\iota_{1}$ and $\iota_{2}$ have to have disjoint images, since so do $f$ and $g$. If $\varphi^{\mathbb{D}}\left(d^{2}, d^{\prime}\right)$ - we use the notation from Figure 7 and consider an essentially binary situation - is undefined, then $\varphi^{\mathbb{C}}\left(c, c^{\prime}\right)$ has to be undefined; however, if $\varphi^{\mathbb{D}}\left(\mathrm{d}, \mathrm{d}^{\prime}\right)$ is defined, then $\varphi^{\mathrm{C}}\left(\mathrm{c}, \mathrm{c}^{\prime}\right)$ has to be defined, too. Because of this contradiction we cannot define a coproduct of $A$ and $B$ in $(E-50 m(\tau)$ nor in $\varepsilon-2$ uom $(\tau)$.

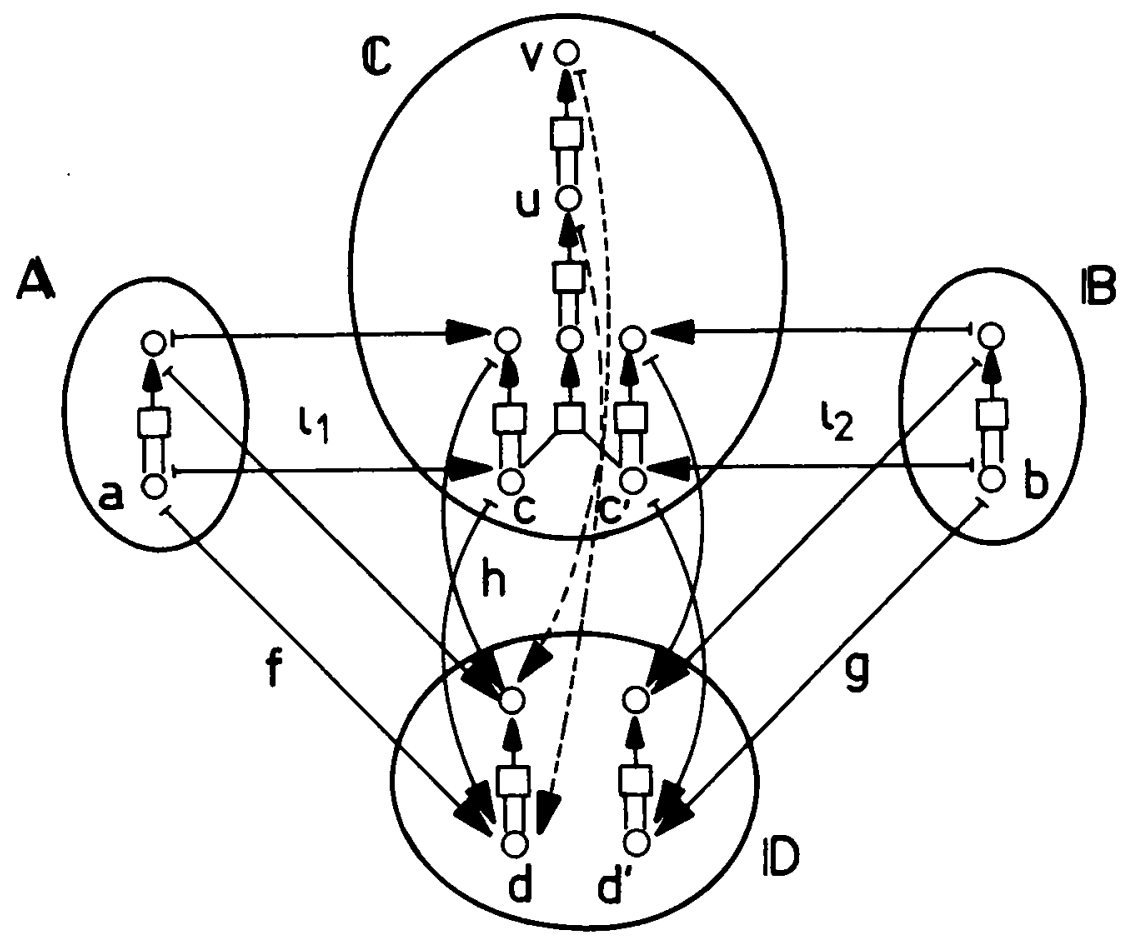

Figure 8: Coproducts exist in general neither in Conf((2)) nor in $5-a$ uom $((2))$.

The argument has to be slightly modified for conf $(\tau)$ and $\tau$-Muom $(\tau)$ : Let $A, \mathbb{B}, f$ and $g$ be given as in Figure 7 or Figure 8. If one takes as $\mathbb{D}$ the absolutely free completion (see [2], 
section 5.3) of the "disjoint union" of $A$ and $B$, then a possible coproduct $C$ of $A$ and $B$ in $\operatorname{con} f(\tau)$ or $\tau-Q$ uom $(\tau)$ has to contain a copy of it. However, if $\mathbb{D}$ is as depicted in Figure 8 , then there are more than one conformism respectively closed quomorphism $h$ out of $C$ into $D$ such, that $f=h \circ \iota_{1}$ and $g=h \circ l_{2}: h$ may or may not be defined e.g. on 'u and $v$. o

There exist coproducts in $\tau$-Duom $\left((0)_{\varphi \in \Omega}\right)$ : Let $\tau$ be a similarity type which only specifies nullary operation symbols. Because of Remark 1.(iii) one has for each sink $\left(\left(f_{i}: \mathbb{A}_{i}-\rightarrow \mathbb{D}\right)_{i \in I}, \mathbb{D}\right)$ of closed quomorphisms: If $\varphi^{\mathbb{D}}$ exists, then $\varphi^{A}{ }^{A}$ exists and belongs to the domain of $f_{i}$ for each $i \in I ;$ and therefore, if $\varphi^{\mathbb{A}} i$ does not exist for at least one $i \in I$, then $\varphi^{\mathbb{D}}$ must not exist. This motivates the definition in the theorem of the coproduct object $\mathbb{C}$, in particular the treatment of the constants: Even if a constant exists in each $A_{i}$, it must not be defined in the coproduct, if it is identical in at least one $\mathbb{A}_{j}$ with a constant which is not defined in all $\mathbb{A}_{i}$. Because of this definition of the constants in $\mathbb{C}$ all "injections" are closed quomorphisms, and any sink will factor through them.

\section{REFERENCES}

[1] P. Burmeister: Partial algebras - survey of a unifying approach towards a two-valued model theory for partial algebras, Algebra Universalis 15(1982) 306-358.

[2] P. Burmeister: A Model Theoretic oriented Approach to Partial Algebras. (Introduction to Theory and Application of Partial Algebras - Part 1). Akademie Verlag, Berlin, 1986.

[3] P. Burmeister, B. Wojdylo: Properties of homomorphisms and quomorphisms between partial algebras. Contributions to General Algebra 5 (Proc. Salzburg Conf., 1986) Verlag Hölder-Pichler-Tempski, wien, Verlag B.G.Teuber, stuttgart, 1987. 
[4] P. Burmeister, B. Wojdylo: The meaning of basic category theoretical notions in some categories of partial algebras. I. Demonstratio Mathematica 25(1992) 583-602.

[5] H. Herrlich, G.E. Strecker: Category Theory - An Introduction. Allyn and Bacon, 1973 (2 ed.: Heldermann-Verlag).

[6] A. G. Kurosch: Gruppentheorie I. Akademie-Verlag, 1970.

[7] S. MacLane: Categories for the Working Mathematician. Springer-Verlag, 1971.

[8] B. Wojdylo: Categories of quasi-algebras. N. Copernicus University, Torun, Preprint No. 2, 1972.

FACHBEREICH MATHEMATIK, AG ALLG. ALGEBRA, TECHNISCHE HOCHSCHULE DARMSTADT SchloBgartenstr. 7, D-6100 DARMSTADT, GERMANY;

DEPARTMENT OF MATHEMATICS, N. COPERNICUS UNIVERSITY, ul. Chopina 12/18 PL-87-100 TORUN, POLAND

Received February 4, 1991. 